\title{
First report of Puccinia vincae in Australia
}

\author{
J. Kruse ${ }^{1}$ (D) $\cdot$ M. Butt ${ }^{2} \cdot$ J. W. Randles ${ }^{3} \cdot$ R. G. Shivas ${ }^{4} \cdot$ A. R. McTaggart ${ }^{5}$
}

Received: 10 January 2020 / Accepted: 10 February 2020 / Published online: 14 February 2020

(C) Australasian Plant Pathology Society Inc. 2020

\begin{abstract}
The rust fungus Puccinia vincae was found for the first time in Australia on blue periwinkle (Vinca major). Puccinia vincae was confirmed by comparison of the ITS2-LSU region to reference sequences from Europe as well as by morphology.
\end{abstract}

Keywords Apocynaceae $\cdot$ Host specificity $\cdot$ Introduced rust

Blue periwinkle (Vinca major, Apocynaceae) is a garden ornamental and an invasive perennial weed in Australia, where it is regarded as a significant environmental weed in Victoria, South Australia, Western Australia, New South Wales, the ACT and Tasmania (Anon 2008; Weeds of Australia, accessed 9 Dec 2019, http://keyserver.lucidcentral.org:8080/weeds/). There are three species of rust fungi (Pucciniales) that infect Vinca in its native range in Europe, namely Puccinia vincae on Vinca major, P. cribrata on V. minor and P. anatolica on $V$. herbacea (Klenke and Scholler 2015).

Three specimens of rust on V. major were collected from urban gardens and roadsides in South Australia in the spring of 2018 (Table 1). Infected plants of BRIP.

68423 and BRIP 68436 had conspicuous brown, powdery uredinia and telia on the abaxial surfaces of shoots of young leaves that were smaller, thicker and lighter than healthy

J. Kruse

julia.kruse1@gmx.de

1 Centre for Crop Health, Institute for Agriculture and the Environment, University of Southern Queensland, Toowoomba, Queensland 4350, Australia

2 Plant Health and Biosecurity, SARDI, Urrbrae, South Australia 5064, Australia

3 School of Agriculture, Food and Wine, University of Adelaide, Waite Campus, Urrbrae, South Australia 5064, Australia

4 Queensland Plant Pathology Herbarium, Department of Agriculture and Fisheries, Dutton Park, Queensland 4102, Australia

5 Queensland Alliance for Agriculture and Food Innovation, The University of Queensland, Ecosciences Precinct, Dutton Park, Queensland 4102, Australia leaves (Fig. 1, a-c). Urediniospores were ellipsoid, oval or globose, echinulate, 28-35 × 18-26 $\mu \mathrm{m}$ (Fig. 1, d \& e, central). Teliospores were 2-celled, with longitudinal stripes and warts, 37-46 × 21-25 $\mu \mathrm{m}$ (Fig. 1, d \& e). The collection from Stirling (BRIP 70683) showed conspicuous shoots with small spermogonia on the abaxial surface of smaller and lighter green leaves (Fig. 1, f). Other plants had small yellow spots (Fig. $1 \mathrm{~g}$ ) on healthy looking leaves with dark brown powdery telia on the abaxial surface. The rust was morphologically identical to Puccinia vincae (Hernandez et al. 2002). All specimens were deposited in the Queensland Plant Pathology Herbarium (BRIP).

DNA was extracted from single pustules of four specimens (Table 1, excluding BRIP 70683) using the DNeasy Ultra Clean Microbial Kit (Qiagen, Hilden). One pustule from each specimen was placed in a $2 \mathrm{~mL}$ plastic reaction tube and homogenized in a Tissue Lyser (Qiagen, Hilden) with glass beads $(0.5-6.35 \mathrm{~mm}$ diam.) at $25 \mathrm{~Hz}$ for $90 \mathrm{~s}$. The ITS2 to large subunit (LSU) region of ribosomal DNA(rDNA) was amplified by PCR with high fidelity Phusion ${ }^{\circledR}$ DNA Polymerase (New England Biolabs,MA, USA), using the primers Rust2INV (Aime 2006) and LR6 (Vilgalys and Hester 1990), and annealing conditions of Aime (2006). Unpurified PCR product was cleaned and sequenced by the Australian Genome Research Facility (AGRF, Brisbane). The LSU sequences were deposited in GenBank (Table 1).

We used a phylogenetic species hypothesis to compare Australian rust specimens on Vinca major to sequences of $P$. vincae in GenBank and a specimen of $P$. cribrata collected from Germany (BRIP 69907). LSU sequences that represented three monophyletic groups of Puccinia (Dixon et al. 2010) were downloaded from GenBank (Table 2) and aligned using MAFFT v.7 (Katoh and Standley 2013). The most likely tree 
Table 1 Specimen details for species of Puccinia on Vinca examined in this study

\begin{tabular}{|c|c|c|c|c|c|c|c|c|c|}
\hline Taxon & Acession No. & Host & Location & Lat & Long & Alt. & Date & Collector & GenBankAcession \\
\hline \multirow[t]{4}{*}{$\begin{array}{r}\text { Puccinia } \\
\text { vincae }\end{array}$} & $\begin{array}{l}\text { BRIP } \\
68423\end{array}$ & \multirow[t]{4}{*}{ Vinca major } & Australia, SA, Adelaide & $34^{\circ} 55^{\prime} 49^{\prime \prime} \mathrm{S}$ & $138^{\circ} 37^{\prime} 0^{\prime \prime} \mathrm{E}$ & $50 \mathrm{~m}$ & $24 / 9 / 18$ & J. Kruse & MN322826 \\
\hline & $\begin{array}{l}\text { BRIP } \\
68436\end{array}$ & & Australia, SA, Belair & $34^{\circ} 59^{\prime} 50^{\prime \prime} \mathrm{S}$ & $\begin{array}{l}138^{\circ} 37^{\prime} 57^{\prime \prime} \\
\mathrm{E}\end{array}$ & $\sim 325 \mathrm{~m}$ & $25 / 9 / 18$ & J. Kruse & MN322828 \\
\hline & BRIP 70683 & & Australia, SA, Stirling & $35^{\circ} 0^{\prime} 0^{\prime \prime} \mathrm{S}$ & $\begin{array}{l}138^{\circ} 25^{\prime} 12^{\prime \prime} \\
\mathrm{E}\end{array}$ & $\sim 525 \mathrm{~m}$ & $15 / 10 / 18$ & J. Randles & - \\
\hline & $\begin{array}{l}\text { BRIP } \\
69750\end{array}$ & & $\begin{array}{l}\text { Italy, Puglia, } \\
\text { Martina Franka }\end{array}$ & $\begin{array}{l}40^{\circ} 39^{\prime} \\
17^{\prime} \mathrm{N}\end{array}$ & $17^{\circ} 18^{\prime} 36^{\prime \prime} \mathrm{E}$ & $\sim 400 \mathrm{~m}$ & $22 / 04 / 16$ & J. Kruse & MN322827 \\
\hline $\begin{array}{l}\text { Puccinia } \\
\text { cribrata }\end{array}$ & $\begin{array}{l}\text { BRIP } \\
69907\end{array}$ & Vinca minor & $\begin{array}{l}\text { Germany, Bavaria, } \\
\text { Coburg }\end{array}$ & $\begin{array}{l}50^{\circ} 16^{\prime} \\
43^{\prime \prime} \mathrm{N}\end{array}$ & $10^{\circ} 55^{\prime} 30^{\prime \prime} \mathrm{E}$ & $\sim 350 \mathrm{~m}$ & $17 / 06 / 17$ & J. Kruse & MN322825 \\
\hline
\end{tabular}

Fig. 1 Phylogram obtained from a maximum likelihood search in IQ Tree v.1.7 beta of the large subunit region of ribosomal DNA. aRLT values $(\geq 0.9)$ and ultrafast bootstrap values $(\geq 95 \%)$ from 10,000 replicates above nodes

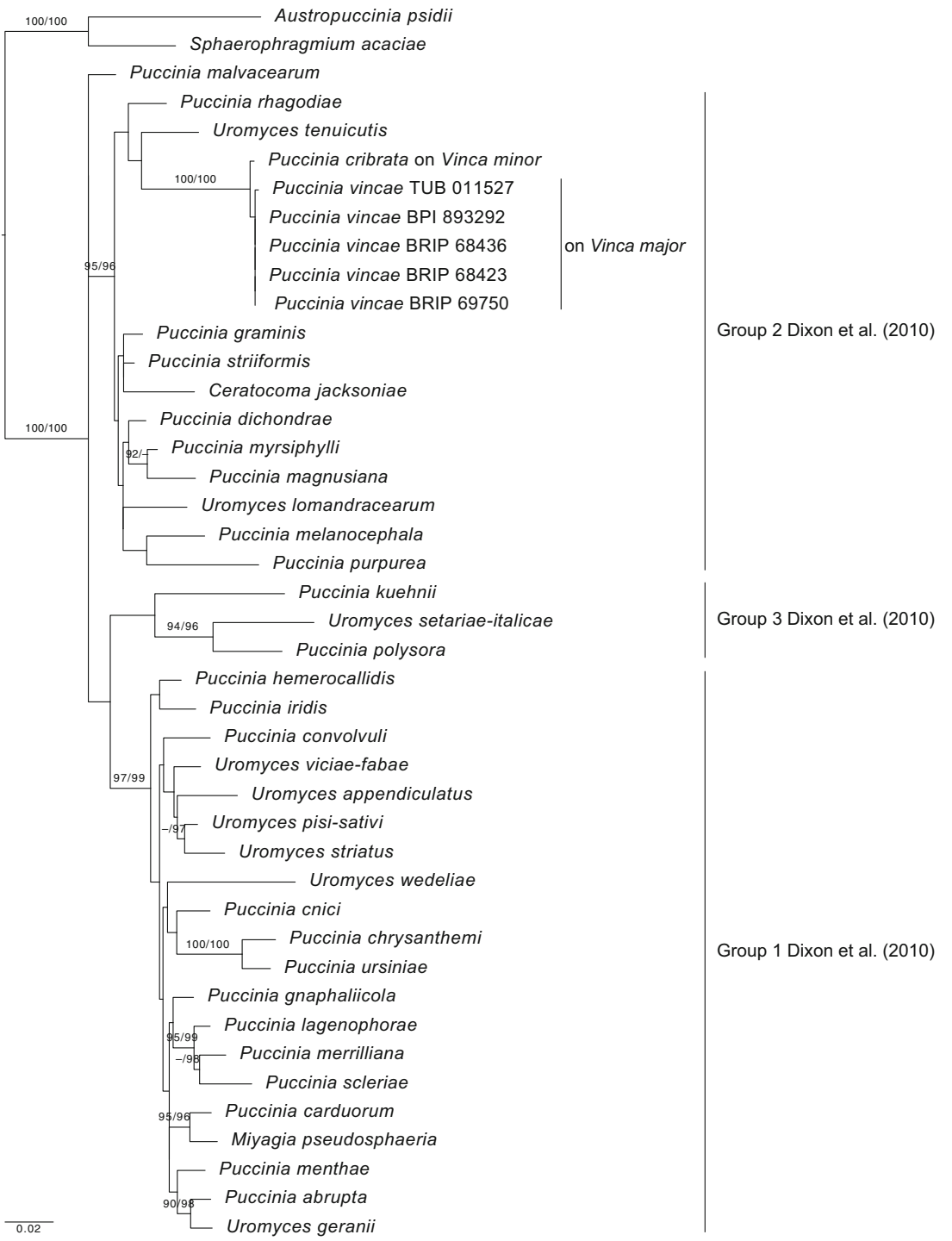


Table 2 Specimen and sequence data of taxa used in phylogenetic analyses

\begin{tabular}{|c|c|c|c|}
\hline Taxon & Source & Herbarium number & GenBank LSU \\
\hline Austropuccinia psidii & Pegg et al. (2014) & BRIP 57793 & KF318449 \\
\hline Ceratocoma jacksoniae & McTaggart et al. (2016b) & BRIP 57762 & KT199394 \\
\hline Miyagia pseudosphaeria & Marin-Felix et al. (2017) & BRIP 59245 & KX999863 \\
\hline Puccinia abrupta & Marin-Felix et al. (2017) & BRIP 59295 & KX999864 \\
\hline Puccinia carduorum & Marin-Felix et al. (2017) & BRIP 60127 & KX999869 \\
\hline Puccinia chrysanthemi & Pedley (2009) & NA & EU816926 \\
\hline Puccinia cnici & Marin-Felix et al. (2017) & BRIP 60111 & KX999872 \\
\hline Puccinia convolvuli & Aime (2006) & BPI 871465 & DQ354512 \\
\hline Puccinia dichondrae & Marin-Felix et al. (2017) & BRIP 60027 & KX999874 \\
\hline Puccinia geitonoplesii & McTaggart et al. (2016a) & BRIP 57603 & KM249861 \\
\hline Puccinia gnaphaliicola & McTaggart et al. (2014a) & BRIP 58451 & KF690703 \\
\hline Puccinia graminis f. sp. tritici & Duplessis et al. (2011) & CRL 75-36-700-3 & NW_003526581.1* \\
\hline Puccinia hemerocallidis & McTaggart et al. (2016a) & BRIP 53476 & KM249855 \\
\hline Puccinia iridis & McTaggart et al. (2016a) & BRIP 56852 & KM249853 \\
\hline Puccinia kuehnii & McTaggart et al. (2016a) & BRIP 59200 & KU296887 \\
\hline Puccinia lagenophorae & McTaggart et al. (2014) & BRIP 57563 & KF690696 \\
\hline Puccinia magnusiana & Dixon et al. (2010) & BPI 879281 & GU058000 \\
\hline Puccinia malvacearum & Matheny PB and Hibbett DS (unpublished) & PBM 2572 & EF561641 \\
\hline Puccinia melanocephala & Dixonet al. (2010) & BPI 878929 & GU058001 \\
\hline Puccinia menthae & Aime (2006) & BPI 871110 & DQ354513 ${ }^{\#}$ \\
\hline Puccinia merrilliana & Marin-Felix et al. (2017) & BRIP 56913 & KX999885 \\
\hline Puccinia myrsiphylli & McTaggart et al. (2016a) & BRIP 57782 & KM249854 \\
\hline Puccinia polysora & Crouch and Szabo (2011) & HSZ1879 & HQ189433 \\
\hline Puccinia purpurea & Marin-Felix et al. (2017) & BRIP 57994 & KX999889 \\
\hline Puccinia rhagodiae & Marin-Felix et al. (2017) & BRIP 60078 & KX999890 \\
\hline Puccinia scleriae & Marin-Felix et al. (2017) & BRIP 56911 & KX999893 \\
\hline Puccinia striiformis & Jin et al. (2010) & HSZ1834 & GQ457306 \\
\hline Puccinia ursiniae & McTaggart et al. (2014a) & BRIP 57993 & KF690705 \\
\hline Sphaerophragmium acaciae & McTaggart et al. (2015) & BRIP 56910 & KJ862350 \\
\hline Uromyces appendiculatus & McTaggart et al. (2014b) & BRIP 60020 & KM249870 \\
\hline Uromyces lomandracearum & McTaggart et al. (2016b) & BRIP 59022 & KM249862 \\
\hline Uromyces pisi-sativi & Marin-Felix et al. (2017) & BRIP 60151 & KX999900 \\
\hline Uromyces setariae-italicae & Marin-Felix et al. (2017) & BRIP 56931 & KX999902 \\
\hline Uromyces striatus & Marin-Felix et al. (2017) & BRIP 61228 & KX999903 \\
\hline Uromyces tenuicutis & Marin-Felix et al. (2017) & BRIP 60012 & KX999904 \\
\hline Uromyces viciae-fabae & McTaggart et al. (2016a) & BRIP 59246 & KM249865 \\
\hline Uromyces wedeliae & Marin-Felix et al. (2017) & BRIP 60000 & KX999907 \\
\hline
\end{tabular}

*whole genome sequence

was searched in IQ Tree v.1.7 beta (Nguyen et al. 2015) with a model test (command -m TEST), 10,000 ultrafast bootstraps (Hoang et al. 2018) and an approximate likelihood ratio test with 10,000 replicates (Guindon et al. 2010). The rust fungi on Vinca occurred in a monophyletic group within a clade of rust fungi on hosts in the Poaceae (Clade II sensu Dixon et al. 2010). Based on a phylogenetic species concept, the
Australian sequences on Vinca major were conspecific with $P$. vincae, which supported the morphological identification. All sequences were identical. Puccinia cribrata (on V. minor) was sister to $P$. vincae (Fig. 2).

This is the first report of $P$. vincae from Australia. Infected periwinkle plants have since been observed at several sites in South Australia between Adelaide and Bridgewater. It is not 
Fig. 2 Symptoms of Puccinia vincae on Vinca major in

Australia: a infected shoots, $\mathbf{b}$ and c uredinia on the abaxial leaf surface, $\mathbf{d}$ and e equatorial and surface view of urediniospores and teliospores (BRIP 68436), $\mathbf{f}$ infected shoots with spermogonia, $\mathbf{g}$ yellow spots of telia on leaves (BRIP 70683). Bar, $10 \mu \mathrm{m}$

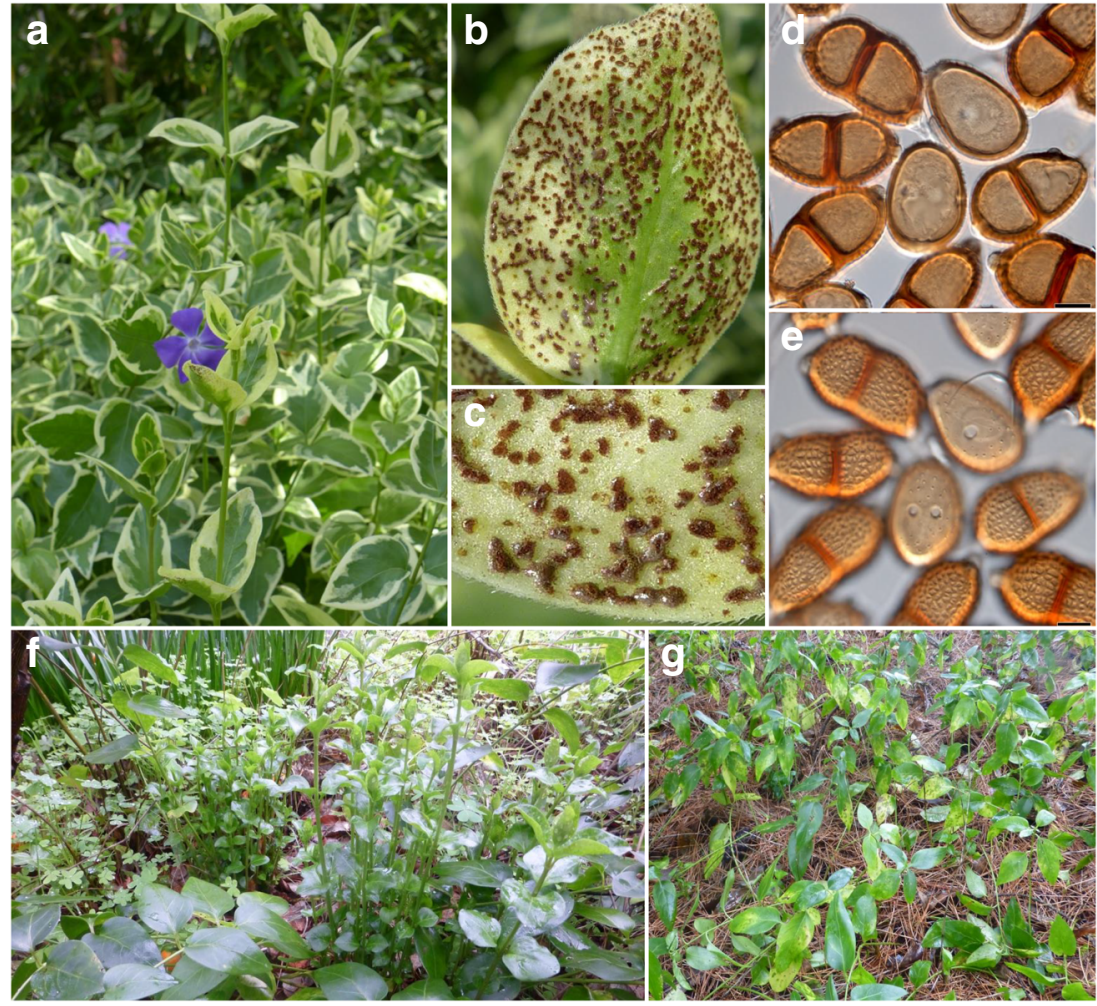

known how and when $P$. vincae was introduced to Australia. Puccinia vincae is widespread on Vinca spp. in Europe and the USA (Hernandez et al. 2002). The rust joins a list of over 100 exotic rusts established in Australia (Shivas et al. 2014).

Acknowledgments We thank Greg Langford of Stirling, and Sue Pederick of Horticulture Pathology SARDI for contributions to the study. This work was partly funded by the Department of the Environment and Energy under the Australian Biological Resources Study (grant number RG18-43).

\section{References}

Aime MC (2006) Toward resolving family-level relationships in rust fungi (Uredinales). Mycoscience 47:112-122

Anon (2008) Weed management guide, managing weeds for biodiversity, Periwinkle (Vinca major). CRC for Australian Weed Management. ISBN: 978-1-920932-71-8 www.weedscrc.org.au

Crouch JA, Szabo LJ (2011) Real-time PCR detection and discrimination of the southern and common corn rust pathogens Puccinia polysora and Puccinia sorghi. Plant Dis 95:624-632

Dixon LJ, Castlebury LA, Aime MC, Glynn NC, Comstock JC (2010) Phylogenetic relationships of sugarcane rust fungi. Mycol Prog 9: 459-468

Duplessis S, Cuomo CA, Lin Y-C, Aerts A, Tisserant E, VeneaultFourrey C, Joly DL, Hacquard S, Amselem J, Cantarel BL, Chiu R, Coutinho PM, Feau N, Field M, Frey P, Gelhaye E, Goldberg J, Grabherr MG, Kodira CD, Kohler A, Kües U, Lindquist EA, Lucas SM, Mago R, Mauceli E, Morin E, Murat C, Pangilinan JL, Park R, Pearson M, Quesneville H, Rouhier N, Sakthikumar S, Salamov
AA, Schmutz J, Selles B, Shapiro H, Tanguay P, Tuskan GA, Henrissat B, Van de Peer Y, Rouzé P, Ellis JG, Dodds PN, Schein JE, Zhong S, Hamelin RC, Grigoriev IV, Szabo LJ, Martin F (2011) Obligate biotrophy features unraveled by the genomic analysis of rust fungi. Proc Natl Acad Sci 108:9166-9171

Guindon S, Dufayard JF, Lefort V, Anisimova M, Hordijk W, Gascuel O (2010) New algorithms and methods to estimate maximumlikelihood phylogenies: assessing the performance of PhyML 3.0. Syst Biol 59:307-321

Hernandez JR, Palm-Hernandez ME, Tidwell T (2002) First report of Puccinia vincae on Vinca spp. in California. Plant Dis 86:75

Hoang DT, Chernomor O, von Haeseler A, Minh BQ, Vinh LS (2018) UFBoot2: improving the ultrafast bootstrap approximation. Mol Biol Evol 35:518-522

Jin Y, Szabo LJ, Carson M (2010) Century-old mystery of Puccinia striiformis life history solved with the identification of Berberis as an alternate host. Phytopathology 100:432-435

Katoh K, Standley DM (2013) MAFFT multiple sequence alignment software version 7: improvements in performance and usability. Mol Biol Evol 30:772-780

Klenke F, Scholler M (2015) Pflanzenparasitische Kleinpilze. Bestimmungsbuch für Brand-, Rost-, Mehltau-, Flagellatenpilze und Wucherlingsverwandte in Deutschland, Österreich, der Schweiz und Südtirol. Springer Berlin Heidelberg, p 1172

Marin-Felix Y, Groenewald JZ, Cai L, Chen Q, Marincowitz S, Barnes I, Bensch K, Braun U, Camporesi E, Damm U, De Beer ZW, Dissanayake A, Edwards J, Giraldo A, Hernández-Restrepo M, Hyde KD, Jayawardena RS, Lombard L, Luangsa-ard J, McTaggart AR, Rossman AY, Sandoval-Denis M, Shen M, Shivas RG, Tan YP, van der Linde EJ, Wingfield MJ, Wood AR, Zhang JQ, Zhang Y, Crous PW (2017) Genera of phytopathogenic fungi: GOPHY 1. Stud Mycol 86:99-216

McTaggart AR, Geering ADW, Shivas RG (2014a) The rusts on Goodeniaceae and Stylidiaceae. Mycol Prog 13:1017-1025 
McTaggart AR, Geering ADW, Shivas RG (2014b) Uredinopsis pteridis and Desmella aneimiae, the first rust fungi (Pucciniales) reported on ferns (Pteridophyta) in Australia. Aust Plant Dis Notes 9:149

McTaggart AR, Doungsa-ard C, Geering ADW, Aime MC, Shivas RG (2015) A co-evolutionary relationship exists between Endoraecium (Pucciniales) and its Acacia hosts in Australia. Persoonia 35:50-62

McTaggart AR, Shivas RG, Doungsa-ard C, Weese TL, Beasley DR, Hall $\mathrm{BH}$, Metcalf DA, Geering ADW (2016a) Identification of rust fungi (Pucciniales) on species of Allium in Australia. Australas Plant Pathol 45:581-592

McTaggart AR, Shivas RG, van der Nest MA, Roux J, Wingfield BD, Wingfield MJ (2016b) Host jumps shaped the diversity of extant rust fungi (Pucciniales). New Phytol 209:1149-1158
Nguyen L-T, Schmidt HA, von Haeseler A, Minh BQ (2015) IQ-TREE: a fast and effective stochastic algorithm for estimating maximumlikelihood phylogenies. Mol Biol Evol 32:268-274

Pedley KF (2009) PCR-based assays for the detection of Puccinia horiana on chrysanthemums. Plant Dis 93:1252-1258

Pegg GS, Giblin FR, McTaggart AR, Guymer GP, Taylor H, Ireland KB, Shivas RG, Perry S (2014) Puccinia psidii in Queensland, Australia: disease symptoms, distribution and impact. Plant Pathol 63:10051021

Shivas RG, Beasley DR, McTaggart AR (2014) Online identification guides for Australian smut fungi (Ustilaginomycotina) and rust fungi (Pucciniales). IMA Fungus 5:195-202

Vilgalys R, Hester M (1990) Rapid genetic identification and mapping of enzymatically amplified ribosomal DNA from several Cryptococcus species. J Bacteriol 172:4239-4246 